\title{
Design of High Throughput Scheduled Mesh Networks: A Case for Directional Antennas
}

\author{
Skanda N. Muthaiah*, Aravind Iyer ${ }^{\dagger}$, Aditya Karnik ${ }^{\ddagger}$ and Catherine Rosenberg* \\ * Department of Electrical and Computer Engineering \\ University of Waterloo, Waterloo, ON, Canada \\ Email: s2nagara@engmail.uwaterloo.ca, catheece.uwaterloo.ca \\ ${ }^{\dagger}$ School of Electrical and Computer Engineering \\ Purdue University, West Lafayette, IN, USA \\ Email iyerav@ecn.purdue.edu \\ ${ }^{\ddagger}$ General Motors - India Science Laboratory \\ International Technology Park, Bangalore, India \\ Email: aditya.karnik@gm.com
}

\begin{abstract}
Scheduled wireless mesh networks (WMNs) represent an important paradigm in the development of high speed wireless access networks. As a consequence of $[1]$, it can be shown that the maximum throughput of a single gateway $n$ node WMN is upper-bounded by $\frac{1}{n}$, normalized with respect to the highest available data-rate. The situation is actually worse since with conventional omni-directional antennas, the achievable throughput is considerably lower at low powers. This paper makes a case for smart (directional) antennas. For the same power, smart antennas can provide higher range, and therefore shorter paths, as compared to omni-directional antennas. For the same range, smart antennas can operate at lower powers, reducing the interference, and thereby improving the spatial reuse. Although they cannot change the fundamental throughput limit of $\frac{1}{n}$, smart antennas can achieve significantly higher throughput at lower powers, These insights are demonstrated through several numerical examples using the framework of [1].
\end{abstract}

\section{INTRODUCTION}

Wireless mesh networks (WMNs) represent an important paradigm for the development of high speed wireless access networks, with upcoming standards like IEEE 802.16 providing mechanisms for coordinated network control and scheduled network operation. However, in order to harness the full potential of the scheduled mesh network architecture, it is important to understand the fundamental limits on the achievable throughput of a network, and how these limits are affected by physical layer parameters and capabilities. As a consequence of the optimization and computational framework of [1], it can be shown that the maximum throughput of a single gateway $n$ node mesh network is upper-bounded by $\frac{1}{n}$, normalized with respect to the highest available data-rate ${ }^{1}$. For instance, with an operating data-rate of $100 \mathrm{Mbps}$ (expected in IEEE 802.16), a 36 node, 1 gateway WMN can achieve a maximum throughput of $100 / 36 \mathrm{Mbps}$ or $2.8 \mathrm{Mbps}$. This indicates the limitations of building very large mesh networks.

Further, with the use of conventional omni-directional antennas, this upper-bound is achieved for very high values of

\footnotetext{
${ }^{1}$ In this work and in [1], it is assumed that all the nodes, including the gateway, are equipped with a single wireless interface.
}

transmit power. For low to moderate powers the achievable throughput turns out to be considerably lower. In general, the use of omni-directional antennas results in poor range for a given transmit power, thereby leading to longer paths, and higher relaying load on the links close to the gateway. Further, since omni-directional antennas radiate uniformly in all directions, for a given range, they result in a considerable amount of interference. The increased interference results in fewer links being scheduled simultaneously affecting spatial reuse and results in reduced throughput This paper makes a case for smart directional antennas. By smart directional antennas, or simply smart antennas, we refer to antenna systems which (i) radiate power not in all directions, but confined within a certain angle of a particular direction, and which (ii) have the ability to orient their beams electronically and therefore quickly, in any particular direction. Some practical examples of smart antennas include switch-beam antennas and phasedarray based antennas.

From a physical layer perspective, smart antennas clearly have certain advantages over conventional omni-directional antennas, and other mechanically steerable directional antennas:

1) Unlike omni-directional antennas, smart antennas confine their radio power in a directed beam, thus saving power and improving range.

2) Unlike mechanically steerable antennas, smart antennas require very little time and power, to change the orientation of their beams.

From a networking perspective, these benefits translate into the following gains:

1) For the same power, smart antennas can provide higher range, and therefore shorter paths, lowering the relaying burden on the links close to the gateway.

2) For the same range, smart antennas can operate at lower powers, and reduce the interference, thereby improving the spatial reuse.

3) In a scheduled WMN, the ability to steer antenna beams helps ensure the alignment of transmitter and receiver 
beams, thus maximizing the gains from directionality. Although smart antennas cannot change the fundamental throughput limit of $1 / n$, they can alleviate interference, and enable efficient operation at lower powers, and over wider areas.

We demonstrate these insights by means of several numerical examples utilizing the optimization and computational framework of [1]. In what follows, we survey some related work in Section II. The optimization framework of [1] is briefly introduced in Section III, followed by a discussion of the antenna models used in this work. Section IV points out the limitations of using omni-directional antennas, and Section $\mathrm{V}$ discusses how these limitations may be addressed by the use of smart antennas. Relevant technological issues are discussed in Section VI. Finally, we conclude the paper in Section VII.

\section{RELATED WORK}

The body of work exploring the impact of using smart antennas to improve the capacity of WMNs, typically falls into two categories, viz., (i) works that study asymptotic capacity scaling [3]-[5], and (ii) works proposing efficient medium access control (MAC) protocols [7]-[10]. In [3], the asymptotic capacity of a random network under an ideally sectorized directional antenna model, is shown to scale as $\Theta\left(\sqrt{n \log ^{3} n}\right)$, assuming the beamwidth can be made arbitrarily small, and that receivers can decode multiple non-overlapping beams simultaneously. Interestingly, despite having such sophisticated directional antennas at one's disposal, the capacity improvements are only of the order $\Theta\left(\log ^{2} n\right)$ over the Gupta-Kumar bound [6]. In [5], the Gupta-Kumar bound has been extended to the case of smart antennas modeled using a simple flattopped antenna model, a phased array model and an adaptive array antenna model. Although the capacity scaling is shown to essentially remain the same, the authors note that by scaling antenna parameters such as number of antenna elements, the capacity could be improved, but not in all cases. Capacity scaling results in terms of antenna beamwidth have been derived in [4], where throughput is shown to improve by a factor of $2 \pi / \sqrt{\alpha \beta}$ where $\alpha$ and $\beta$ are the transmit and receive antenna beamwidths, respectively.

Some MAC protocols proposed for smart antennas include [8] for ideally sectorized antennas, [7] for switched-beam antennas, and [9] for phased array antennas. [8] proposes a multi-hop RTS MAC protocol (MMAC) for directional antennas. Through simulations on a $5 \times 5$ grid and a random network for different instances of routes, the authors show a throughput increase of upto $400 \%$ over IEEE 802.11. In [10], the authors propose a Directional Busy Signal MAC (DBSMA) protocol and show significant improvements in throughput, over the Directional MAC (DMAC) protocol proposed in [8]. Their protocol also uses a more general directional antenna model than the ideally sectorized antenna model used in [8].

We take a different approach to study the impact of smart antennas. Our aim is to study the throughput-capacity of an arbitrary wireless network employing smart antennas, not in an asymptotic scaling sense, or by proposing random access protocols, but by accurately computing the maximum throughput achievable by the network, under scheduled network operation, through detailed modeling of physical layer parameters and capabilities.

\section{Motivation, Optimization And Computational FRAMEWORK, AND MODELS}

In this section, we lay down the background for the rest of the paper. We start by motivating and introducing single gateway scheduled WMNs, followed by the optimization and computational framework of [1]. We also introduce the physical channel model, and the antennas models.

\section{A. Single gateway scheduled WMNs}

In this work, we consider single gateway scheduled WMNs where the term scheduled refers to the fact that these networks operate by means of precisely scheduling different subsets of links, rather than having individual links or nodes use random access. Single gateway scheduled WMNs are likely to be deployed for cellular back-haul or for broadband wireless access. There are two advantages to using scheduling in WMNs. Firstly, the throughput performance of scheduled WMNs is expected to be much superior to random access based WMNs. Secondly, in case of smart antennas, the ability to steer beams can be combined with the precise knowledge of link transmission schedules, to align the transmitter and receiver beams of every link, thus maximizing the gains from directionality. This is in contrast with using smart antennas with random access, where researchers have experienced some difficulty in fully exploiting directionality [10]. The data traffic in these networks is typically either upload traffic where the nodes send data to the (access) gateway (and eventually to the Internet), or download traffic which originates from the gateway, and is destined to a node. Typically, there is no traffic destined from one node to another.

\section{B. Optimization Framework}

The aim of the optimization and computational framework in [1] is to characterize and compute the capacity and optimal configuration of a network specified in terms of the set of nodes, and a set of flows given as source-destination pairs. The notion of capacity is that of max-min throughput, i.e., our aim is to maximize the minimum flow throughput that can be achieved by appropriately configuring the network. As we shall see, a configuration completely specifies the set of links, their physical layer parameters, the flow routes and the link activation schedules. In [1], two formulations of the above problem are presented, a routing-based one, and a schedulingbased one. We only review the scheduling formulation in this paper.

Let us begin by introducing some notation. Let $\mathcal{N}$ be the set of nodes numbered $1,2, \ldots, N$, and let $\mathcal{L}$ be the set of directed links numbered $1,2, \ldots, L$. Here, without loss of generality, the set of links can be chosen to be the set of edges in the complete directed graph on the set of nodes in 
the network. The feasibility of these links depends on the set of physical layer parameters used. In order to model the capability of modern radios to use different transmit powers and modulation schemes, the set of links can be thought of as a set of "virtual" links. Here for every physical link (from the complete directed graph on the set of nodes, say), we assume multiple virtual links operating between the link's origin and destination, each virtual link corresponding to a particular combination of transmit power and modulation scheme. For more details, please refer to [1].

For the purposes of this work, we abstract the physical layer parameters into link capacities for each (virtual) $\operatorname{link}^{2}$, and the set of "independent" sets of links. Based on the underlying physical layer parameters, if a link $l$ is feasible, then it is assigned a data-rate $c_{l}$ depending on its modulation scheme. If the link $l$ is not feasible for the combination of power and modulation being used on it, $c_{l}$ is taken to be zero. The set of independent sets of links, $\mathcal{I}$, characterizes the simultaneous operation of sets of links, based on the interference caused by the links to one another. Note that these independent sets are not the familiar graph-theoretic independent sets, but merely sets of links which can operate simultaneously, with each link maintaining a satisfactory BER (bit-error rate).

We assume that a given BER specification translates into an SINR (signal-to-interference-and-noise) requirement for every link. Specifically, we assume that a link $l$ requires an SINR of at least $\beta_{l}$ (which depends on the modulation scheme used on l) for successful operation. Thus, denoting a subset of links by an $L$-dimensional vector $x$, where $x_{l}=1$ implies link $l \in x$, $x_{l}=0$ implies $l \notin x$, we can describe the set of independent sets $\mathcal{I}$ as:

$$
\mathcal{I}=\left\{x: \frac{G_{l l} P_{l}}{N_{0}+\sum_{l^{\prime}, l \neq l^{\prime}} G_{l^{\prime} l} P_{l^{\prime}} x_{l^{\prime}}}>\beta_{l} x_{l}, \forall l \in \mathcal{L}\right\}
$$

where $P_{l}$ denotes the power used on link $l, G_{l l}$ denotes the gain from the transmitter to the receiver of link $l, G_{l^{\prime} l}$ denotes the gain from the transmitter of $l^{\prime}$ to the receiver of $l$, and $N_{0}$ denotes the noise power in the operating frequency band. The gains $G_{\{. .\}}$are assumed to be known and fixed. For more details on modeling interference using BER or SINR, please refer to [2].

The set of flows is denoted by $\mathcal{F}$, and the flows are numbered $1,2, \ldots, M$. A given flow $f$ is associated with a source node $f_{s}$ and a destination node $f_{d}$. For a given node $i$, the set of outgoing links is denoted by $\mathcal{L}_{i}^{O}$, and the set of incoming links by $\mathcal{L}_{i}^{I}$. For a given link $l$, the set of independent sets it belongs to is denoted by $\mathcal{I}_{l}$. A link activation schedule (or simply, link schedule) is denoted by $\alpha=\left\{\alpha_{k}, k \in \mathcal{I} \mid \sum_{k \in \mathcal{I}} \alpha_{k}=1\right\}$, where $k$ is a generic independent set, and $\alpha_{k}$ represents the fraction of time the independent set $k$ is active. The flow variables $x_{l}^{f}$ denote the amount of traffic of flow $f$ on link $l$. Finally, $\lambda_{f}$ denotes the throughput of flow $f$, and $\lambda$ denotes the minimum throughput.

\footnotetext{
${ }^{2}$ In the rest of the paper, the term "link" would refer to a virtual link, unless stated otherwise.
}

Under the above notation, the problem of capacity and optimal configuration, can be posed as the following optimization problem:

$$
\begin{array}{cl}
\max \lambda & \\
\sum_{l \in \mathcal{L}_{i}^{o}} x_{l}^{f}-\sum_{l \in \mathcal{L}_{i}^{I}} x_{l}^{f} & = \begin{cases}0 & i \notin\left\{f_{s}, f_{d}\right\} \\
\lambda_{f} & i=f_{s} \\
-\lambda_{f} & i=f_{d}\end{cases} \\
\sum_{f \in \mathcal{F}} x_{l}^{f} \leq c_{l} \sum_{k \in I_{l}} \alpha_{k} & l=1, \ldots, N, f=1, \ldots, M \\
\sum_{k \in \mathcal{I}} \alpha_{k}=1 & \\
0 \leq \lambda \leq \lambda_{f} & f=1, \ldots, L
\end{array}
$$

where the first of set of constraints are to ensure flow conservation and the second set of constraints are the link capacity constraints. The other constraints just ensure that $\alpha$ is a valid schedule, and that $\lambda$ is the minimum flow throughput.

\section{Physical Layer Models}

The models used for the physical channel and for the antennas, get reflected in the set of independent sets $\mathcal{I}$, via the gains $G_{l l}$ and $G_{l^{\prime} l}$. Now we discuss how these gains are modeled. We take the channel gains to be given by isotropic path loss. Specifically, the channel gain between two points which are a distance $d$ apart, is given by $\left(\frac{d}{d_{0}}\right)^{-\eta}$ where $d_{0}$ is the far-field crossover distance, and $\eta$ is the path loss exponent. Omni-directional antennas are modeled as isotropic radiators with a gain of unity. Smart antennas are modeled as directional antennas whose beams can be steered on a per-independent set basis to get perfect alignment between the transmitter and receiver beams of each link in the given independent set. The directional beams are modeled as that of a parabolic antenna with a circular aperture. However, we ignore back lobes and side lobes. The main lobe is characterized by a gain $\Gamma$ and a beamwidth $\theta$ which can be computed from the aperture $a$ as follows:

$$
\Gamma=\epsilon\left(\frac{\pi a}{w}\right)^{2} ; \quad \theta=\frac{70 w}{a}
$$

where $w$ is the operating wavelength [11]. Along the axis of the beam, the perceived gain with respect to an omnidirectional antennas is $\Gamma$, while at an angle of $\phi$ with respect to the axis of the beam, the gain is lower by a factor of $16^{\left(\frac{\phi}{\theta}\right)^{2}}$ [12]. Thus, the gains $G_{l l}$ and $G_{l^{\prime} l}$ would be calculated as follows. For omni-directional antennas:

$$
G_{l l}=\left(\frac{d_{l l}}{d_{0}}\right)^{-\eta} ; G_{l^{\prime} l}=\left(\frac{d_{l^{\prime} l}}{d_{0}}\right)^{-\eta}
$$

For smart antennas:

$G_{l l}=\Gamma^{2}\left(\frac{d_{l l}}{d_{0}}\right)^{-\eta} ; G_{l^{\prime} l}=\left(\frac{\Gamma^{2}}{16^{\left(\phi_{l^{\prime} l}^{t x} / \theta\right)^{2}} 16^{\left(\phi_{l l^{\prime}}^{r x} / \theta\right)^{2}}}\right)\left(\frac{d_{l^{\prime} l}}{d_{0}}\right)^{-\eta}$

where $d_{l l}$ is the transmitter-receiver separation of link $l, d_{l^{\prime} l}$ is the distance from the transmitter of $l^{\prime}$ to the receiver of $l$, $\phi_{l^{\prime} l}^{t x}$ is the angle of the antenna beam of the transmitter of $l^{\prime}$, and $\phi_{l l^{\prime}}^{r x}$ that of the antenna beam of the receiver of $l$. Both angles are with respect to the line joining the transmitter of $l^{\prime}$ to the receiver of $l$ (refer to Figure 1). 


\section{Computational Approach}

It has been shown in [1] that in general, computing the maxmin throughput of an arbitrary network is an NP-hard problem. However, it is also shown in [1] that it is possible to exactly solve the problem numerically, under certain assumptions, for the case of omni-directional antennas. This is because under the assumptions, it is possible to establish a bound on the size of the maximum independent set in the network. Then, the technique used in [1] for the case of omni-directional antennas, is that of enumerating the set of independent sets, by using this bound. This is accomplished by only checking the "independence" of all subsets of $\mathcal{L}$, of a size smaller than the bound.

For this approach to work in the case of smart antennas, we need to derive a bound on the size of the maximum independent set in the network. Instead, we take the following approach. Rather than attempting to solve the problem exactly, we solve it approximately, by enumerating all independent sets of a size smaller than a complexity parameter, we term MAXISET. By increasing the value of MAXISET, the accuracy of our results can be improved. There are two advantages to using this approach: (i) the throughput we obtain through this approach is clearly always a lower bound on the actual achievable throughput; and (ii) the parameter MAXISET introduces a tradeoff between complexity and performance. The higher the value of MAXISET the more accurate the results, at the cost of increased computational complexity of enumerating larger sized subsets. We do not claim optimality of the numerical results we present, for smart antennas, but we feel this is a reasonable approach because even with modest values of MAXISET, we are able to demonstrate considerable gains in using smart antennas.

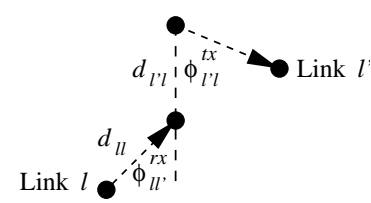

Fig. 1. Variables in the gain calculations for Omni-directional and Smart antennas; arrows indicate the receivers of the links.

\section{Fundamental Limits ANd Limitations of CONVENTIONAL ANTENNAS}

In this section, we show that the throughput of a single gateway $n$ node mesh network is upper bounded by $1 / n$ normalized with respect to the highest operating data-rate. Thereafter, we look at the limitations of using conventional omni-directional antennas. For the sake of simplicity, we consider only upload traffic in the rest of the paper. Specifically, we associate each node with a flow originating from that node, and destined to the gateway. Clearly any other combination of traffic flows, whether upload, download or node-to-node, can be handled by our optimization framework.
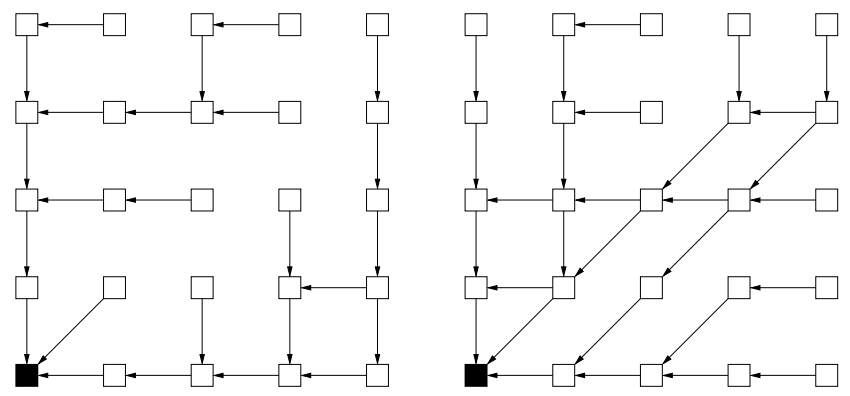

Fig. 2. Optimal Routing for a $5 \times 5$ grid employing: (i) omni-directional antenna with transmit power $-7.75 \mathrm{dBm}$ (left); (ii) smart antennas with beamwidth $52^{\circ}$ and transmit power $-22.48 \mathrm{dBm}$ (right).

\section{A. Upper bound on the throughput of a single gateway mesh network}

Consider the problem formulation in (2) for a single gateway mesh network. Consider one flow associated with every node (other than the gateway) destined to the gateway. Let $c$ be the maximum data-rate of any link. Let 0 denote the gateway node, and let $n$ be the number of nodes (not including the gateway). Then we have the following theorem.

Theorem 4.1: The throughput of a single gateway $n$ node mesh network is upper bounded by $c / n$ where $c$ is the maximum data-rate of any link in the network.

Proof: Since $\lambda$ is the minimum flow throughput, we have

$$
\begin{aligned}
\lambda & \leq \frac{\sum_{f \in \mathcal{F}} \lambda_{f}}{n} \\
& =\frac{\sum_{f \in \mathcal{F}} \sum_{l \in \mathcal{L}_{0}^{I}} x_{l}^{f}}{n} \\
& \leq \frac{\sum_{l \in \mathcal{L}_{0}^{I}} c_{l} \sum_{k \in \mathcal{I}_{l}} \alpha_{k}}{n} \\
& \left.\leq \frac{c}{n} \sum_{l \in \mathcal{L}_{0}^{I}} \text { (Fraction of time Link } l \text { is active }\right) \\
& =\frac{c}{n}(\text { Fraction of time Node } 0 \text { is receiving }) \\
& \leq \frac{c}{n}
\end{aligned}
$$

where the second equality follows from flow conservation at the gateway node 0 , the third inequality follows from the link capacity constraints for all the links incoming to the gateway node 0 , the fourth inequality follows since $c$ is the maximum data-rate of any link in the network, and finally, we have the upper bound since the sum of the fractions of time links incoming to the gateway are active, is the fraction of time the gateway is receiving, which is less than unity.

Clearly, if the transmit power of each node enables it to communicate directly with the gateway at the data-rate $c$, then this simple single-hop configuration achieves the upper-bound of Theorem 4.1, albeit at very high transmit powers.

\section{B. Performance of conventional omni-directional antennas}

Now we look at the performance of a single gateway WMN equipped with omni-directional antennas. While the maximum normalized capacity has been shown to be $1 / n$, the capacity achieved at moderate to low powers, turns out to 
be considerably lower. We consider the single gateway WMN depicted in Figure 2. It is a network of 24 nodes and a gateway arranged in a $5 \times 5$ grid, with the gateway placed at the bottom left corner. The separation between adjacent nodes along the vertical and horizontal edges is $8 \mathrm{~m}$. All the nodes use the same transmit power and modulation scheme. The modulation scheme has an SINR threshold of $10 \mathrm{~dB}$ (corresponding to a BER of $10^{-6}$ ). We use the computational technique discussed earlier in Section III to generate the numerical results. The variation of the capacity as a function of the transmit power is depicted in Figure 3.

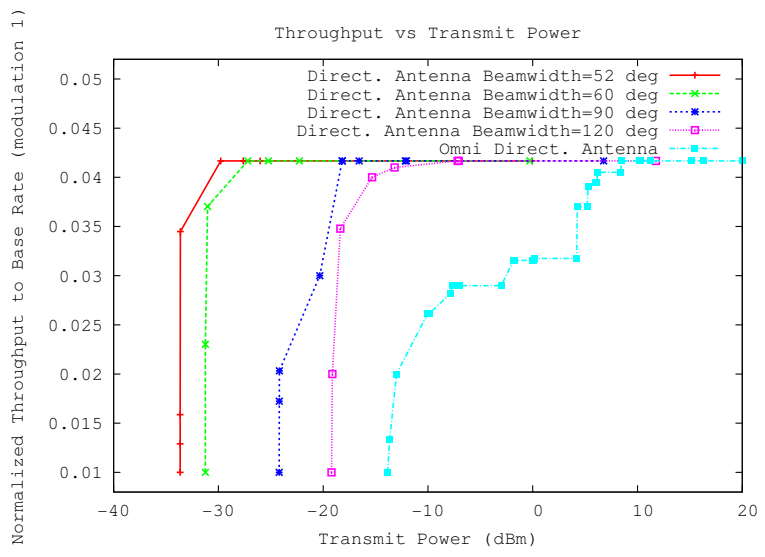

Fig. 3. Variation of $\lambda$ vs Transmit Power (dBm)

Several observations are in order. First observe that the throughput achieved at very low powers when the network just becomes connected, is less than one-fourth of the maximum achievable throughput. As the power is increased, there is a dramatic improvement in throughput to about $60 \%$ of the maximum at around $-8 \mathrm{dBm}$. Beyond this point, the throughput increases quite slowly with transmit power, finally achieving the maximum at a considerably high transmit power of 9 $\mathrm{dBm}$ or so. Figure 2 (left) depicts the optimal routing used by the network at a transmit power of $-7.75 \mathrm{dBm}$. Although the range based on the transmit power is such that diagonal links can be used, only one such link is used in the optimal configuration. Due to the high interference caused by omnidirectional antennas, the routes tend to avoid interference by taking long paths which in turn increase the relaying load on the links incoming to the gateway, throttling the throughput.

This is also well-reflected in Figure 4 which depicts the size of the maximum independent set used in the optimal configuration. Although, larger independent sets become feasible as transmit power increases, it turns out that the size of the maximum independent set used actually reduces beyond a certain transmit power. This figure provides a scheduling perspective, and illustrates the extent of spatial reuse. We see that spatial reuse is only a secondary metric, and can actually reduce even though the throughput is increasing. However, the important point is that the spatial reuse is unable to increase to beyond 5, for any transmit power, due to the interference caused by omni-directional antennas.

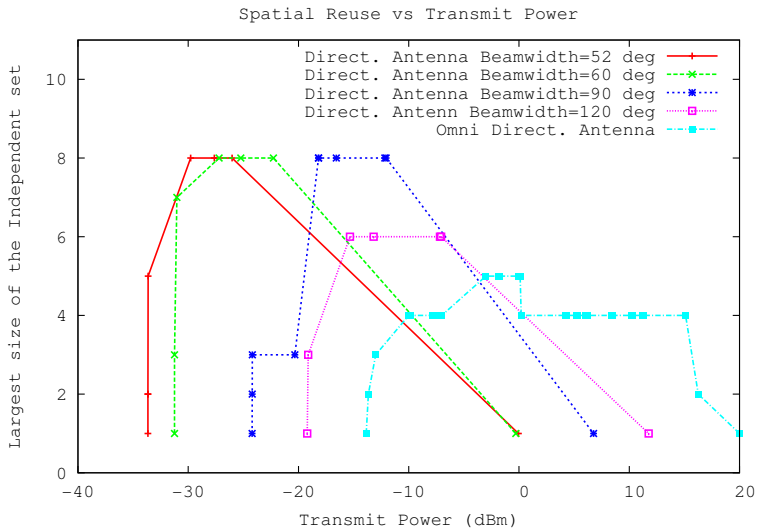

Fig. 4. Variation of Spatial Reuse with Transmit Power (dBm)

\section{A CASE For SMart Antennas}

In this section, we see how the limitations of omnidirectional antennas can be avoided by using smart antennas. Figure 3 depicts the variation of capacity with transmit power, for smart antennas for different values of the beamwidth. It is clear that the maximum throughput achieved by the network is still bounded by $\frac{1}{n}$, even with smart antennas. This is of course in accordance with Theorem 4.1 which was independent of physical layer capabilities. It is also evident from Figure 3 that the capacity increases considerably faster with transmit power, for smart antennas, especially so for smaller beamwidths. Finally, smart antennas achieve the maximum throughput at an overall lower power compared to omni-directional antennas. This is reflected in the fact that the throughput curve for smart antennas, appears to be shifted to the left with respect to that for omni-directional antennas.

In deriving the numerical results for smart antennas, we have fixed the bound on the size of the maximum independent set i.e., MAXISET to be 8 . In spite of choosing a potentially suboptimal set of independent sets, the gains of using smart antennas are apparent from Figure 3. Figure 4 shows the spatial reuse in case of smart antennas, for different beamwidths, to be higher than that for omni-directional antenas. Since MAXISET has been fixed at 8 , a spatial reuse of higher than 8 cannot be observed, although it could be possible in practice.

In Figure 2 (right), the optimal routing for a smart antenna system with beamwidth $52^{\circ}$, is depicted for a transmit power of $-22.63 \mathrm{dBm}$. This transmit power is such that the nodes have a range of $11.413 \mathrm{~m}$, the same range as with a power of -7.75 $\mathrm{dBm}$ with omni-directional antennas. In contrast to Figure 2 (left), here we observe a lot more diagonal links being used in the optimal configuration. This can be attributed to the fact that owing to the directed beams used by smart antennas, they result in much lower interference. Hence, the optimal routes do not really have to "avoid" one another.

As we saw earlier, owing to their directionality, smart antennas provide a gain of $\Gamma$ with respect to omni-directional antennas. Hence, a smart antenna can achieve the same transmission range for significantly lower powers $\left(P \rightarrow \frac{P}{\Gamma^{2}}\right)$. Also for the same power, smart antennas can provide connectivity at increased inter-node separation $\left(D \rightarrow \Gamma^{2 / \eta} D\right)$. This facilitates 
the use of smart antennas at low powers, and over wide areas.

\section{Smart Antennas: Practical And TECHNOLOGICAL ISSUES}

In this work, we considered an ideally sectorized antenna model. We also assumed it is possible to precisely and quickly align the beams of the transmitter and the receiver antenna ensuring maximum gain. In practice, this capability can be achieved by the use of (i) Phased Array Antennas, or (ii) Switched-beam Antennas.

Phased array antennas achieve beam-steering, by constantly changing the excitation phase of the antenna elements that constitute the array. In practice, however, the phase difference between individual array elements determines the overall beamwidth of the antenna array and bears a significant effect on the beamwidth as the beam is steered [11]. The number of elements constituting the antenna array, their arrangement, their relative displacements along with the phase differences, all contribute to the overall radiation pattern of the antenna and in several cases introduce, remove or modify sidelobes and/or backlobes in the radiation pattern [13]. However, the ability of phased array antennas to steer their beams in any direction makes them an ideal choice for WMNs. In fact, all nodes can be designed with the same homogeneous phased array system.

Switched-beam antennas are not capable of precise beamsteering but instead are equipped with a number of directional antenna elements oriented in some pre-defined directions [14]. Switched-beam antennas can electronically switch between these constituent beams, thus exhibiting some degree of beamsteering. These antennas are cost-effective [15] as compared to phased array antennas, and do not exhibit changing radiativity patterns. However, their limited or fixed beam-steering capability makes transmitter-receiver beam-alignment difficult and rigid. This might also require configuring the constituent beams on the nodes, on a per-node basis, depending on the topology.

It is clearly necessary to incorporate realistic smart antenna models, and to deal with technological limitations, in the design of WMNs. However, notwithstanding any of these technological issues, our aim in this paper was to highlight the benefits and advantages of using smart directional antennas. We believe that by offering insights on the use of directional antennas, we have demonstrated their benefits. Incorporating realistic smart antenna models in our design framework is part of our future research agenda.

\section{CONCLUSIONS}

The throughput of a single gateway $n$ node $\mathrm{WMN}$, normalized by the highest operating data-rate, is upper-bounded by $1 / n$. This is a pessimistic result which indicates the infeasibility of building large WMNs. However, the situation is actually worse because with omni-directional antennas, it requires very high transmit powers to achieve this upperbound. With low to moderate powers, the throughput achieved is considerably lower. Thus, there is a clear case for the use of smart antennas. Smart antennas, although cannot change the fundamental throughput bound of $1 / n$, can bring about substantial improvements in the performance at intermediate powers. By directing their power in a narrow beam, smart antennas can enable efficient operation at lower powers and over wider areas. To conclude, smart antennas are clearly the technology of choice for scheduled WMNs.

\section{REFERENCES}

[1] Aditya Karnik, Aravind Iyer and Catherine Rosenberg, "Throughputoptimal Configuration of Wireless Networks", in Proceedings of the Allerton Conference, September 2006.

[2] A. Iyer, C. Rosenberg and A. Karnik, What is the Right Model for Wireless Channel Interference? in Proceedings of the $3^{\text {rd }}$ International Conference on Quality of Service in Heterogeneous Wired/Wireless Networks, QShine 2006.

[3] Christina Peraki and Sergio D. Servetto, "On the Maximum Stable Throughput Problem in Random Networks with Directional Antennas", in Proceedings of ACM Mobihoc 2003, pp 76-87, Maryland, US, 2003.

[4] Su Yi, Yong Pei, and Shivkumar Kalyanaraman "On the Capacity Improvement of Ad Hoc Wireless Networks Using Directional Antennas", in Proceedings of ACM Mobihoc 2003, pp. 108-116, Maryland, US, 2003.

[5] Akis Spyropoulos and Cauligi S. Raghavendra, "Asympotic Capacity Bounds for Ad-hoc Networks Revisited: The Directional and Smart Antenna Cases", IEEE Globecom 2003, Vol 3, pp. 1216-1220, 2003.

[6] Piyush Gupta and P.R.Kumar, "The Capacity of Wireless Networks", IEEE Transactions on Information Theory, Vol. IT-46, no. 2, pp. 388404, March 2000

[7] Sedat Atmaca, Celal Ceken, and Ismail Erturk, "Capacity Enhancement in Wireless Networks using Directional Antennas", World Enformatika Society, Transactions on Engineering, Computing and Technology, pp 174-179, vol 1, 2006

[8] Romit Roy Choudhury, Xue Yang, R. Ramanathan and Nitin H. Vaidya, "On Designing MAC Protocols for Wireless Networks Using Directional Antennas", IEEE Transactions on Mobile Computing, No 5, Vol 5, pp 477-491, May 2006.

[9] Harkirat Singh and Suresh Singh, "Tone based MAC protocol for use with Adaptive Array Antennas", in Proceedings of IEEE WCNC 2004, pp 1246-1251,, vol 1, 2004.

[10] Sunil S. Kulkarni and Catherine Rosenberg, "DBSMA: A MAC Protocol for Multi-hop Ad-hoc Networks with Directional Antennas", in Proceedings of PIMRC 2005, September 2005.

[11] Constantine Balanis, "Antenna Theory: Analysis and Design", John Wiley and Sons, USA, 1997.

[12] Robert A. Nelson, "Antennas: The Interface with Space", Applied Technology Institute (ATI) Space and Communications Training Courses, September 1999. Available at http: //www .aticourses.com/antennas_tutorial.htm

[13] Mineo Takai, J. Martin, A. Ren and Rajive Bagrodia "Directional Virtual Carrier Sensing for Directional Antennas in Mobile Ad Hoc Networks", in Proceedings of ACM Mobihoc 2002, Lausanne, Switzerland

[14] Sheng, W.X., Zhou, J., Fang, D.G. and Gu, Y.C. " Super-resolution DOA estimation in switch beam smart antenna" Proceedings of the 5th International Symposium on Antennas, Propagation and EM Theory, 2000. ISAPE 2000. pp 603-606, Beijing, China

[15] Karmakar, N.C. and Bialkowski, M.E. "Electronically steerable array antennas for mobile satellite communications-a review" Proceedings of the International Conference on Phased Array Systems and Technology, 2000. pp 81-84, Dana Point, CA 